\title{
Sinonasal Teratocarcinosarcoma- A Rare Tumour not so Rarely Misdiagnosed
}

\begin{tabular}{cl}
\multicolumn{1}{c}{ Jayalakshmy P Leelamma ${ }^{\mathbf{1}}$, Bhavya P. Mohan ${ }^{\mathbf{1}}$, Aparna Srinivasan } \\
1. Dept. of Pathology, Government Medical College, Kottayam, Kerala. India
\end{tabular}

Corresponding information: Dr. Jayalakshmy P.L, Assistant professor, Department of pathology, Govt. Medical college Kottayam, KERALA. Ph number:9048149456 e-mail ID: jayafloyyd@gmail.com

Copyright $\odot$ 2017, IRANIAN JOURNAL OF PATHOLOGY. This is an open-access article distributed under the terms of the Creative Commons Attribution-noncommercial 4.0 International License which permits copy and redistribute the material just in noncommercial usages, provided the original work is properly cited.

\section{Introduction}

STCS is a rare and highly malignant tumour with combined features of a teratoma and carcinosarcoma. It was first described by Shanmugaratnam in 1983 as teratoidcarcinosarcoma, consisting of various components of neuroectodermal, epithelial and mesenchymal proliferations of varying maturity (1). Keeping its complex cytoarchitecture in mind, the tumour was later named as sinonasal teratocarcinosarcoma by Heffner et al. in 1984, after their review of 20 cases (2).This histologic heterogeneity, a morphologic hallmark of STCS accounts for numerous diagnostic errors in small biopsies. Of 20 cases reviewed by Heffner, many were mislabelled as adenocarcinoma, olfactory neuroblastoma, fibrosarcoma and rhabdomyosarcoma (2). These patients have dismal prognosis, with frequent recurrences and short mean survival. One case series calculated a two-year disease free survival rate of $28 \%$ and the overall survival rate of $46 \%$ (3).

\section{Case History}

A 48-year-old male presented to ENT (Ear, Nose and Throat) department with complaints of right nasal obstruction for three months. Diagnostic nasal endoscopy revealed a polypoidal mass arising from the skull base extending to right nasal cavity. CT PNS revealed a mass in the right nasal cavity with erosion into the right cribriform plate. MRI (Magnetic Resonance Imaging) scan with contrast (Fig 1A) revealed right nasoethmoidal mass eroding the cribriform plate with intracranial extension on the right side. He underwent incisional biopsy of the mass lesion which showed squamous epithelial nests with peripheral palisading and abundant wet keratin in a fibrous stroma. With these features, the histopathology report was given as craniopharyngioma. Finally, endoscopic excision of the polypoidal mass was performed under general anaesthesia.

We received the specimen at pathology department as multiple nodular grey white soft tissue pieces aggregate measuring $8 \times 7 \times 2 \mathrm{~cm}$. The microscopic examination showed a malignant 
neoplasm composed of epithelial and mesenchymal components. Epithelial component was composed of nests of poorly differentiated cells to well differentiated squamous cells with keratin pearl formation (Fig 1B) and glands filled with mucin (Fig 1C). There were clear squamous cells forming nests. The mesenchymal components showed loose connective tissue with chondromyxoid appearance. In addition to osteoid formation (Fig 1D).

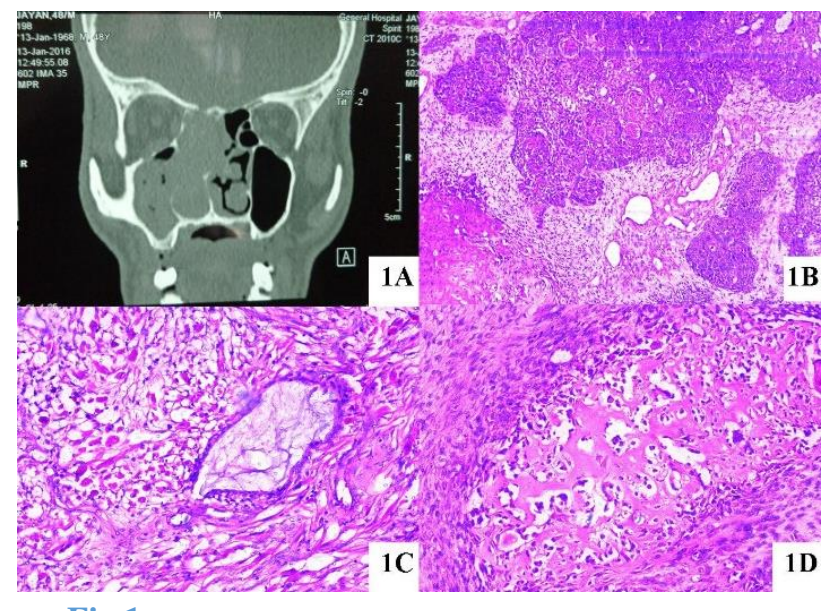

Fig 1.

A: MRI shows right nasoethmoidal mass with erosion of cribriform plate.

B: Areas of well and poorly differentiated squamous cell carcinoma (H \& E, 200X magnification).

C: Dilated gland surrounded by mesenchymal component (H and E, 200X magnification).

D. A focus of osteoid formation ( $\mathrm{H}$ and $\mathrm{E}, 200 \mathrm{X}$ magnification).

There were areas of rhabdomyosarcomatous differentiation (Fig 2A) and immature cartilage. Islands of primitive neuroepithelial tissue with true rosettes and pseudorosettes (Fig 2B) were also seen. These findings were consistent with STCS. Immunohistochemistry showed expression of vimentin in the mesenchymal component, desmin (Fig 2C) in rhabdomyosarcomatous areas, neuronspecific enolase (Fig 2D) in primitive neuroepithelial component, cytokeratin and epithelial membrane antigen (Fig 3A) in the epithelial component and CD99 (Fig 3B) in primitive neuroepithelium. Hence, a final diagnosis of sinonasal teratocarcinosarcoma was rendered.

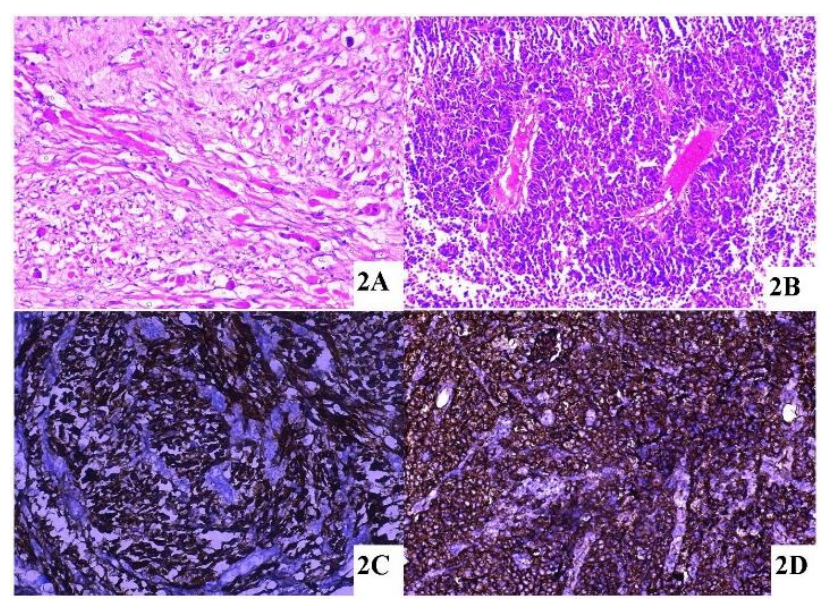

Fig 2.

A: Rhabdomyosarcomatous differentiation ( $\mathrm{H}$ and $\mathrm{E}$, 200X magnification).

B: Neuroectodermal component with perivascular pseudorosettes (H \& E, 200X magnification).

$\mathrm{C}$ : Rhabdomyosarcomatous area is positive for Desmin (IHC, 200X magnification).

D: Neuroectodermal component is NSE positive (IHC, 200X magnification).

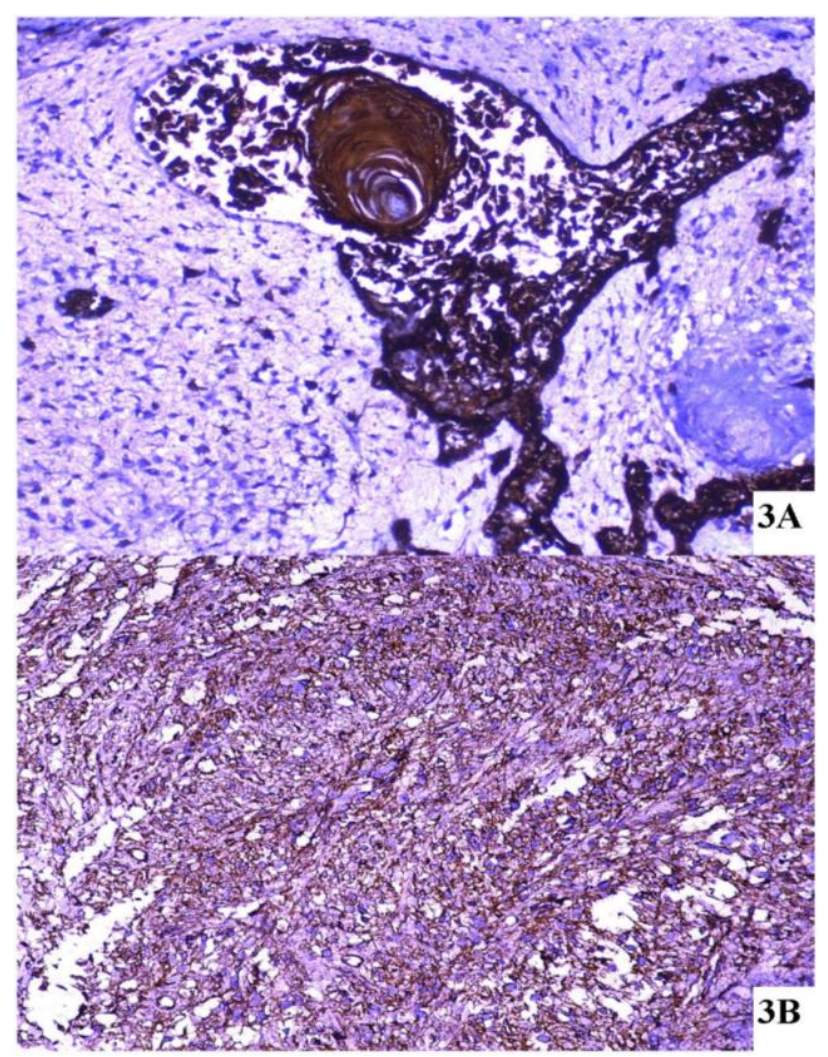

Fig 3.

A: Squamous epithelium is EMA positive (IHC, 200X magnification).

B. Neuroectodermal component is CD 99 positive (IHC, 200X magnification). 
$\mathrm{He}$ was then referred for postoperative chemotherapy and radiation treatment. The patient took five cycles of chemotherapy and was currently receiving radiation therapy at the time of wiring the manuscript.

\section{Discussion}

STCS is thought to arise from the pluripotent olfactory epithelial cells in the nasal cavity.

However, some ambiguity exists regarding the cells of origin. Most authors agree that a pluripotent progenitor cell with multidirectional differentiation capabilities is the most likely case giving rise to the tissue heterogeneity $(4,5)$.

Morphologically, these tumours are bulky and reddish friable masses. On microscopy, they show a diversity of tissue types with combination of epithelial, mesenchymal and neuroectodermal components with variable degrees of maturity (46).The epithelial components include benign and malignant squamous epithelium and glandular epithelium. Among these, immature fetal type squamous epithelium is very frequent, seen in a significant proportion of cases $(5,6)$. The mesenchymal components may be formed by benign or malignant fibromyxoid, chondroid, osteoid or myoid elements and the neuroectodermal component shows neural rosette formation with or without fibrillary background (4-6).

The present case had characteristic features of STCS like atypical squamous epithelium, wellformed glands, rhabdomyosarcomatous areas, fibromyxoid areas and primitive neuroepithelium with pseudorosettes. Areas of chondroid and smooth muscle differentiation were visibly absent.

Since this tumour shows a variegated microscopic appearance and situated in the midline, differential diagnoses are vast and include poorly differentiated squamous cell carcinoma, sarcoma, malignant craniopharyngioma, small cell carcinoma and sinonasal undifferentiated carcinoma (6).Coexistence of carcinomatous and sarcomatous elements rules out the above mentioned differential diagnoses. The undifferentiated round cells characteristic of STCS may show varying degrees of neuroectodermal differentiation mimicking olfactory neuroblastoma, but are unique because of the presence of overt epithelial differentiation and true neoplastic mesenchymal components $(6,7)$. Similarly, teratoma with malignant transformation can resemble STCS except for the fact that teratomas mainly occur in the reproductive system and other parts of the body, whereas the location of STCS is well defined. Furthermore, teratoma lacks immature squamous cell nests (clear cell nests) and does not exhibit carcinosarcomatous features $(6,7)$. It is different from true carcinosarcoma which consists of a single malignant epithelial and a single malignant mesenchymal component, whereas STCS has one or many epithelial and mesenchymal components (7).

Limited biopsy samples may therefore fail to establish the diagnosis as it happened in the present case. Immunohistochemical staining is dependent on the cell type. Epithelial components have positive results for cytokeratin and epithelial membrane antigen; neuroepithelial components have positive findings for neuron-specific enolase, CD99, chromogranin, synaptophysin, glial fibrillary acidic protein and S-100 protein; mesenchymal components have positive result for vimentin and may be reactive for myogenic markers or smooth muscle actin depending on cell types (7).

These highly malignant tumours initially present with relatively benign complaints of recurrent epistaxis (53.52\% of cases) and nasal obstruction (61.97\% of cases) (4). Review of literature showed that these symptoms presented within a short mean duration of 102 days, which could be attributed to the aggressive nature of the tumour. Most common sites involved are the nasal cavity and paranasal sinuses. Bone erosion and intracranial extension were also found in $24.19 \%$ and $20.96 \%$ of the reported cases respectively (4). The present case also had bone erosion and intracranial extension. The average survival as reported by Heffner and Hyams is 1.7 years (2). Analysis of the available literature on STCS showed a strong male predominance with an average age of presentation at about 51.75 years $(7,8)$.

Although there is no defined treatment protocol, recent reports confirm that multimodality treatment for STCS, with maximal safe surgical resection followed by radiotherapy and or chemotherapy, is the most effective modality to date (9). 


\section{Conclusion}

STCS is a rare, unique entity, which often poses a diagnostic challenge, particularly on small biopsy samples or partially removed tumour specimens. Aggressive sampling for histopathological examination is necessary to avoid erroneous diagnosis. Furthermore, in view of very poor prognosis, multimodality treatment in the form of postoperative radiotherapy and chemotherapy needs to be standardized. Very aggressive follow-up is necessary to detect early recurrences. Early diagnosis and management before regional spread can give a better prognosis.

\section{Conflict of interest}

The authors declared no conflict of interest.

\section{References}

1. Shanmugaratnam K, Kunaratnam N, Chia KB, Chiang GS, Sinniah R. Teratoid

Carcinosarcoma of the paranasal sinuses. Pathology 1983; 15(4): 413-9.

2. Heffner DK, Hyams VJ. Teratocarcinosarcoma (malignant teratoma?) of the nasal cavity and paranasal sinuses, a clinicopathologic study of 20 cases. Cancer 1984;53(10):2140-54

3. Budrukkar A, Agarwal JP, Kane S, Siddha M, Laskar SG, Pai P, et al.Management and clinical outcome of sinonasal teratocarcinosarcoma: single institution experience. J LaryngolOtol 2010; 124(7): 739-43.

4. Smith SL, Hessel AC, Luna MA, Malpica A, Rosenthal DI, El-Naggar AK. Sinonasal teratocarcinosarcoma of the head and neck: a report of 10 patients treated at a single institution and comparison with reported series. Arch Otolaryngol Head Neck Surg 2008; 134(6): 592-5.

5. Batsakis JG, El-Naggar AK, Luna MA. Teratomas of the head and neck with emphasis on malignancy. Ann OtolRhinolLaryngol 1995; 104(6): 496-500.

6. Yang S, Sun R, Liang J, Zhou Z, Zhou J, Rui J. Sinonasal teratocarcinosarcoma: A clinical and pathological analysis. Int J Surg Pathol 2013 ;21(1):37-43.

7. Pai SA, Naresh KN, Masih K, Ramarao C, Borges AM. Teratocarcinosarcoma of the paranasal sinuses: a clinicopathologic and immunohistochemical study. Hum Pathol 1998; 29(7): 718-22.

8. Kane SV, Karpate AA, Bal M, Juvekar SL, Pai PS. Chemotherapy-induced neuronal maturation in sinonasal teratocarcinosarcoma- a unique observation. Head Neck Pathol 2009; 3(1): 31-6.

9.Misra P, Husain Q, Svider PF, Sanghvi S, Liu JK, Eloy JA.Management of sinonasal teratocarcinosarcoma: A systematic review. Am J Otolaryngol2014;35(1):5-11.

How to Cite This Article

Leelamma J, Mohan B, Srinivasan A. Sinonasal Teratocarcinosarcoma- A Rare Tumour not so Rarely Misdiagnosed. Iran J Pathol. 2018; 13(1): 85-88. 